\title{
Effects of dietary replacement of soybean meal with dried distillers grains with solubles on the microbiota occupying different ecological niches in the rumen of growing Hu lambs
}

Junshi Shen ${ }^{1,2+}$, Zhipeng $\mathrm{Li}^{1,3+}$, Zhongtang $\mathrm{Yu}^{4}$ and Weiyun Zhu ${ }^{1,2^{*}}$

\begin{abstract}
Background: Diet has a profound impact on the rumen microbiota, and the impact can vary among the different rumen ecological niches (REN). This study investigated the effects of dietary replacement of soybean meal (SBM) with dried distillers grains with solubles (DDGS) on the rumen microbiota occupying different REN of growing Hu lambs. After a 9-week feeding trial, 6 lambs from each dietary treatment (SBM vs. DDGS-based diets) were slaughtered for sample collection. The microbiota of the rumen solid, liquid, and epithelium fractions was examined using amplicon sequencing analysis of bacterial $16 \mathrm{~S}$ rRNA gene, functional prediction, and qPCR.

Results: No interaction of dietary protein source (PS) and REN were detected for virtually all the measurements made in this study. The DDGS substitution resulted in very limited influence on bacterial community structure. However, the metabolic pathways predicted from 16S rRNA gene sequences varied greatly between SBM- and DDGS-based diets. The populations of rumen total bacteria, fungi, sulfate-reducing bacteria (SRB), and methanogens were not influenced by DDGS substitution, but the population of protozoa was reduced. The bacterial communities in rumen solid (RS) and liquid (RL) were similar in taxonomic composition but were different in relative abundance of some taxa. In contrast, the bacterial composition and relative abundance of rumen epithelium (RE) were greatly distinct from those of the RS and the RL. In alignment with the bacterial relative abundance, the metabolic pathways predicted from $16 \mathrm{~S}$ rRNA genes also varied greatly among the different REN. The populations of total bacteria, protozoa, and methanogens attached to the RE were smaller than those in the RS and RL, and the fungal population on the rumen epithelium was smaller than that in the RS but similar to that in the RL. On the contrary, the SRB population on the RE was greater than that in the RS and RL. (Continued on next page)
\end{abstract}

\footnotetext{
* Correspondence: zhuweiyun@njau.edu.cn

${ }^{\dagger}$ Junshi Shen and Zhipeng Li contributed equally to this work.

'Laboratory of Gastrointestinal Microbiology, Jiangsu Key Laboratory of

Gastrointestinal Nutrition and Animal Health, College of Animal Science and

Technology, Nanjing Agricultural University, Nanjing 210095, China

${ }^{2}$ National Center for International Research on Animal Gut Nutrition, Nanjing

Agricultural University, Nanjing 210095, China

Full list of author information is available at the end of the article
}

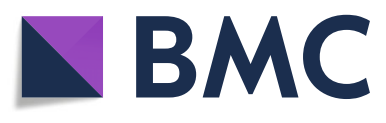

(c) The Author(s). 2020 Open Access This article is licensed under a Creative Commons Attribution 4.0 International License, which permits use, sharing, adaptation, distribution and reproduction in any medium or format, as long as you give appropriate credit to the original author(s) and the source, provide a link to the Creative Commons licence, and indicate if changes were made. The images or other third party material in this article are included in the article's Creative Commons licence, unless indicated otherwise in a credit line to the material. If material is not included in the article's Creative Commons licence and your intended use is not permitted by statutory regulation or exceeds the permitted use, you will need to obtain permission directly from the copyright holder. To view a copy of this licence, visit http://creativecommons.org/licenses/by/4.0/. The Creative Commons Public Domain Dedication waiver (http://creativecommons.org/publicdomain/zero/1.0/) applies to the data made available in this article, unless otherwise stated in a credit line to the data. 
(Continued from previous page)

Conclusions: Substitution of SBM with DDGS had greater impact to the protozoa than to the other microbes, and the microbial community structure and functions at different REN are distinct and niche-adapted.

Keywords: Distillers dried grains with solubles, Growing lamb, Microbiota, Ruminal ecological niche, Soybean meal

\section{Background}

Rumen microbiota determines animal health and production performance, and a better understanding of this complex and diverse microbiota, especially the relationship between the structure and function under different dietary conditions can lead to innovative interventions to improve animal productivity [1]. Dietary changes can shift rumen microbiota with respect to its composition and structure and fermentation patterns [2, 3]. Dried distillers grains with solubles (DDGS) is an abundant byproduct of ethanol production from grains (primarily corn), which are high in crude protein $(\mathrm{CP})$, ether extract (EE) and energy content $[4,5]$, and low in cost [6]. It has been widely used as a substitute for corn and soybean meal (SBM) in ruminant production [7, 8]. Several studies have evaluated the impact of feeding DDGS on rumen bacterial community in steers [9-11] and dairy cattle [12, 13]. However, the influences of DDGS addition on rumen microbial community structure and function in growing lambs remain poorly understood. In our previous study using growing lambs, substituting DDGS for SBM in an isonitrogenous diet increased dietary EE, neutral detergent fiber (NDF) and acid detergent fiber (ADF) contents, and significantly altered rumen fermentation parameters such as volatile fatty acid (VFA) and ammonia concentrations [5].

Within the rumen ecosystem, the environments create three different ruminal ecological niches (REN), namely the solid, liquid, and epithelium $[14,15]$. The three REN have different community structure [15-18] and rumen function $[17,19]$. Previous studies have compared the bacterial community occupying the three REN using polymerase chain reaction-denaturing gradient gel electrophoresis (PCR-DGGE) [17, 20-22], cloning [16, 21] or high-throughput sequencing $[15,18,19,23-25]$. To our knowledge, most of these studies using dairy cattle or steers as experimental animals, and only one study, which used PCR-DGGR [17], investigated the bacterial community occupying the different REN of growing lambs. Recently, we also evaluated the changes of the bacterial communities at the three different REN of growing $\mathrm{Hu}$ lambs in response to dietary urea supplementation [26]. However, most of the studies focused only on the community structure and function of rumen bacteria, without considering the eukaryotes [27]. This study, by integrating real-time quantitative polymerase chain reaction (qPCR), high-throughput sequencing, and functional prediction, investigated the effect of dietary replacement of SBM with DDGS on community structure and function of the microbiota at the three different REN of growing $\mathrm{Hu}$ lambs.

\section{Methods}

Animals, diets and experimental design

The experimental design, diets, and management have been reported previously [5]. Briefly, The DDGS replaced all the SBM and a portion of the ground corn in the diets. The feeding trial was conducted for 10 weeks, with the first 1 week for adaptation followed by 9 weeks of dietary treatment. At the end of week 9, six lambs each were randomly selected only from the SBM-Control and the DDGS-Control groups and slaughtered for sample collection.

\section{Sample collection}

On days 6 and 7 of week 9 , the six lambs each selected from the SBM-Control and DDGS-Control were slaughtered at $4-6 \mathrm{~h}$ after morning feeding. The whole rumen contents of each $\mathrm{Hu}$ lamb were first homogenized, mixed, and then strained through four layers of cheesecloth to separate the rumen liquid (RL) and the rumen solid (RS) fractions. Approximately $30 \mathrm{~mL}$ each of liquid and the solid fraction was collected into a sterilized tube and immediately stored in liquid nitrogen. To collect the rumen epithelial (RE) samples, the rumen walls were rinsed with cold sterile saline solution three times after the removal of the rumen contents. Epithelial tissue samples from an approximately $8 \mathrm{~cm}^{2}$ area of the rumen epithelium were scraped using a sterilized glass slide and stored in liquid nitrogen immediately after collection. The RS, RL, and RE samples were stored at $-80^{\circ} \mathrm{C}$ until further analysis.

\section{DNA extraction}

Metagenomic DNA of the rumen solid, liquid, and epithelium samples was extracted using the bead-beating and phenol-chloroform extraction method as previously described [28]. The quality of the DNA extracts was visually checked using electrophoresis on $1.2 \%$ agarose gel (w/v) containing Goldview ${ }^{\text {mix }}$ (SaiBaiSheng, Shanghai, China), and the DNA concentration of each sample was determined using a Nanodrop 2000 (Thermo Fisher Scientific, Inc., Madison, USA). The DNA samples were stored at $-20^{\circ} \mathrm{C}$ until analyses. 


\section{Illumina sequencing of $16 \mathrm{~S}$ rRNA gene amplicons and data analysis}

The V3-V4 hypervariable regions of the 16S rRNA gene were amplified using primers 338F (5'-ACTCCTACGG GAGGCAGCA-3') and 806R (5'-GGACTACHVGGG TWTCTAAT-3 ). Unique barcodes were added to the 5 end of both primers for multiplexing. PCR products were verified on agarose gel $(2 \%, \mathrm{w} / \mathrm{v})$, and the expected bands were each extracted and purified using the AxyPrepDNA Gel Extraction Kit (Axygen Biosciences, CA, USA). The concentrations of the purified DNA amplicons were each quantified using a QuantiFluor ${ }^{\circ}$ dsDNA kit (Promega, Madison, WI, USA). Amplicons from different samples were mixed in equal ratio and sequenced using the $2 \times 300$ paired-end kit on an Illumina MiSeq platform. The raw sequence reads were deposited into the NCBI Sequence Read Archive (SRA) database under the accession number PRJNA565493.

Raw fastq files were de-multiplexed, quality-filtered, and analyzed using QIIME 1.9.1 [29]. Operational taxonomic units (OTUs) were de novo clustered with a 97\% sequence similarity cutoff using UPARSE (version 7.1 http://drive5.com/uparse/), and possible chimeric sequences were identified and removed using UCHIME [30]. The most abundant sequence within each OTU was selected as the 'representative sequence' and was taxonomically classified based on the SILVA database (version 128) [31]. A PH Lane mask supplied by QIIME was used to remove the hypervariable regions from the aligned sequences. FASTTREE [32] was used to create a phylogenetic tree of the representative sequences for each sample. Sequences identified as of chloroplasts or mitochondria were removed before further analysis. Alpha diversity measurements including observed OTUs, Chao1 richness estimate, and Shannon diversity index, as well as Good's coverage, were calculated using QIIME 1.9.1 [29]. Principal coordinates analysis (PCoA) was performed based on weighted UniFrac distance and Bray-Curtis dissimilarity to reveal overall differences in prokaryotic communities among the rumen solid, liquid and epithelial fractions from SBM and DDGS groups (SBM-RS, SBM-RL, SBM-RE, DDGS-RS, DDGS-RL, and DDGS-RE). The functional profiles of the rumen microbiota from different REN of the growing $\mathrm{Hu}$ lambs were predicted from the 16S rRNA gene data using Tax4Fun [33]. The functional profiles were summarized at hierarchy level 2 of Kyoto Encyclopedia of Genes and Genomes (KEGG) pathways.

\section{Quantitative real-time PCR analysis}

The PCR primers used for real-time qPCR of total bacteria [34], fungi [34], protozoa [35], methanogens [36], and SRB [37] are listed in Table S1. Real-time PCR was performed on a StepOnePlus system (Applied Biosystems, California,
USA) using the SYBR Premix Ex Taq dye (Takara Bio Inc.). Copies of $16 \mathrm{~S}$ rRNA gene (total bacteria), $18 \mathrm{~S}$ rRNA gene (fungi and protozoa), methyl coenzyme-M reductase alpha subunit gene ( $m c r A$, for methanogens), and dissimilatory sulfite reductase alpha subunit gene (dsrA, for SRB) in each sample was quantified in three technical replicate against respective standards, which were purified PCR products of known length and concentration. The absolute abundance of each microbial population was expressed as copies of the target gene/g of samples.

\section{Statistical analyses}

Analysis of similarity (ANOSIM) was used to assess the statistical significance of the PCoA analysis of overall microbiota across the treatments. The real-time PCR data were $\log$ transformed to improve normality. Residual analysis was used to determine if a transformation of variables was needed. If needed, cubic root transformations were performed. All data (absolute abundance quantified by qPCR, alpha diversity measurements, relative abundances of microbial populations at the phylum and genus levels, and the relative abundance of level 2 KEGG pathways) were analyzed using the MIXED procedure of SAS version 9.4 (SAS Institute Inc., Cary, NC). The model included PS and REN as fixed effects, with block as random effects. Degrees of freedom were calculated using the KenwardRoger option. The mean separation test was performed using the Tukey multiple range test. Differences were considered statistically significant at $P \leq 0.05$.

\section{Results}

\section{Effect on bacterial alpha diversity}

Across all 36 samples, a total of 1,275,639 quality-checked $16 \mathrm{~S}$ rRNA gene sequences were obtained. On average, each sample had at least 35,434 sequences. Greater than 99.3\% depth coverage was achieved for all the treatments (Table 1). There were no interactions $(P \geq 0.06)$ between PS and REN for OTU numbers, Chao 1, or Shannon index. None of the alpha diversity measurements was affected $(P \geq 0.36)$ by dietary PS. The number of OTUs and Chao 1 richness estimate in the RS and the RL fractions were similar $(P>0.05)$, which were higher $(P<0.05)$ than in the RE fraction. In contrast, The Shannon index was higher $(P>0.05)$ in the RS than in the RL and the RE fraction.

\section{Effect on overall bacteria}

The PCoA analysis, based on either Weighted UniFrac or Bray-Curtis dissimilarity, indicated a clear separation between the epithelium and the liquid or solid fraction, but no dietary PS effects were observed (Fig. 1). Moreover, the samples from the RS and the RL fractions clustered closely together, indicating very similar community structures. These findings were further supported by the ANOSIM based on the Bray-Curtis distance $(P<0.001$; Figure S1). 
Table 1 Effect of replacing soybean meal with dried distillers grains with solubles on the alpha diversity measurements of ruminal microbiota (at 3\% dissimilarity level) of different ruminal ecological niches of growing Hu lambs

\begin{tabular}{|c|c|c|c|c|c|c|c|c|c|c|}
\hline \multirow[t]{2}{*}{ Item } & \multicolumn{3}{|c|}{ Protein source (PS) } & \multicolumn{4}{|c|}{ Ruminal ecological niche (REN) } & \multicolumn{3}{|c|}{$P$-value } \\
\hline & SBM & DDGS & SEM & $\overline{\text { Solid }}$ & Liquid & Epithelial & SEM & PS & REN & $P S \times R E N$ \\
\hline Coverage, $\%$ & 99.3 & 99.3 & 0.03 & 99.3 & 99.3 & 99.3 & 0.03 & 0.36 & 0.14 & 0.43 \\
\hline Observed OTUs & 650.6 & 667.3 & 27.50 & $711.5^{\mathrm{a}}$ & $694.3^{\mathrm{a}}$ & $571.1^{\mathrm{b}}$ & 31.02 & 0.57 & $<0.01$ & 0.33 \\
\hline Chao 1 & 820.1 & 837.1 & 26.66 & $870.6^{\mathrm{a}}$ & $861.0^{\mathrm{a}}$ & $754.2^{\mathrm{b}}$ & 31.80 & 0.63 & 0.02 & 0.48 \\
\hline Shannon index & 4.39 & 4.39 & 0.089 & $4.73^{\mathrm{a}}$ & $4.34^{\mathrm{b}}$ & $4.11^{\mathrm{b}}$ & 0.109 & 0.98 & $<0.01$ & 0.06 \\
\hline
\end{tabular}

${ }^{\text {a-b } M e a n s ~ w i t h ~ d i f f e r e n t ~ s u p e r s c r i p t s ~ w i t h i n ~ a ~ r o w ~} \operatorname{differ}(P<0.05)$

\section{Effect on bacteria at phylum and genus levels}

In total 19 bacterial phyla were identified among all the treatments, with Bacteroidetes, Firmicutes, Proteobacteria, Spirochaetes, Fibrobacteres, and Actinobacteria being the six most predominant phyla, each of which was represented by more than $0.5 \%$ of the total sequences in at least one treatment (Table 2). No interaction $(P \geq 0.59)$ of PS with REN was detected with respect to any of the bacterial phyla. None of the bacterial phyla was affected $(P \geq 0.11)$ by dietary PS, but all were greatly influenced by REN. The relative abundance of Bacteroidetes (52.15-55.01\%) in the RS and the RL was higher $(P<0.01)$ than that in the RE fraction $(27.41 \%)$. On the contrary, the relative abundance of Proteobacteria (0.64-0.93\%) and Spirochaetes (0.76$2.45 \%)$ in the RS and the RL was lower $(P<0.01)$ than that in the RE fraction (18.06\% and 6.23\%). The relative abundance of Fibrobacteres was higher (1.16\%) in the RS than in the RL and the RE fractions $(0.12-0.30 \%)$. The relative abundance of Firmicutes (41.41-45.63\%) and Actinobacteria $(0.65-0.85 \%)$ were not influenced $(P \geq 0.55)$ by REN.

A total of 238 bacterial genera were identified among all the treatments, but only 49 of them were each represented by more than $0.5 \%$ of the total sequences in at least one treatment (Table 3 ) and they were regarded as the "major genera". No interaction $(P \geq 0.13)$ of PS with REN was detected on any of the bacterial abundance at the genus level except for the genera Pseudobutyrivibrio and Suttonella $(P<0.01)$. The relative abundance of Ruminococcaceae UCG-005 was higher $(P<0.01)$ for the lambs receiving SBM than those fed DDGS, while the relative abundance of other major bacterial genera was not influenced $(P \geq 0.08)$ by PS. In contrast, 39 out of the 49 major genera were significantly different $(P<$ 0.05) among the three REN. Compared with the RS and

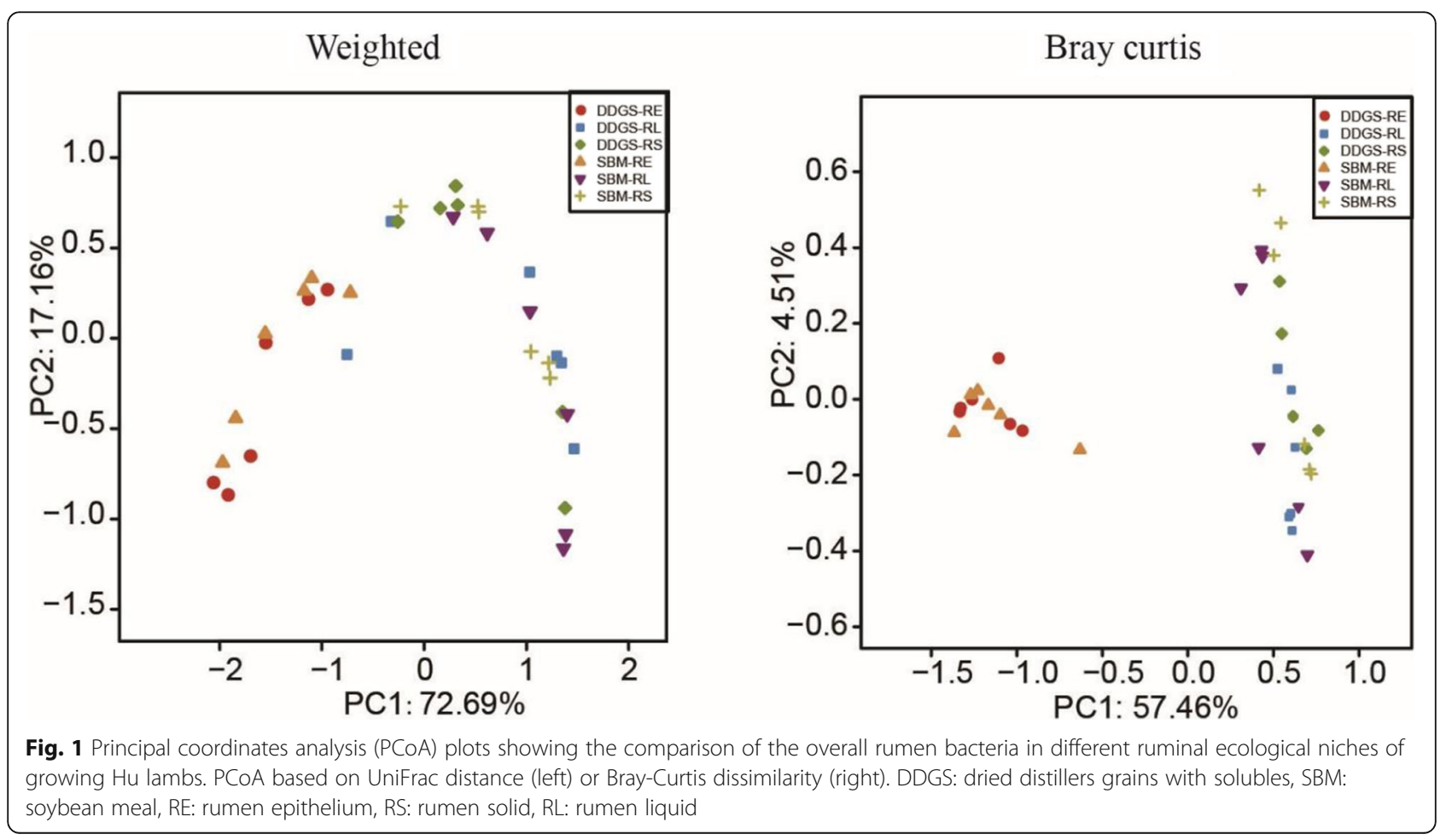


Table 2 Effect of replacing soybean meal with dried distillers grains with solubles on relative abundance of major ruminal bacterial phylum (each with a relative abundance $\geq 0.5 \%$ in at least one treatment) in different ruminal ecological niches of growing $\mathrm{Hu}$ lambs

\begin{tabular}{|c|c|c|c|c|c|c|c|c|c|c|}
\hline \multirow[t]{2}{*}{ Phylum } & \multicolumn{3}{|c|}{ Protein source (PS) } & \multicolumn{4}{|c|}{ Ruminal ecological niche (REN) } & \multicolumn{3}{|c|}{$P$-value } \\
\hline & SBM & DDGS & SEM & Solid & Liquid & Epithelial & SEM & PS & REN & $\mathrm{PS} \times \mathrm{REN}$ \\
\hline Bacteroidetes & 47.80 & 41.92 & 2.779 & $52.15^{\mathrm{a}}$ & $55.01^{a}$ & $27.41^{b}$ & 3.404 & 0.15 & $<0.01$ & 0.83 \\
\hline Firmicutes & 40.65 & 45.21 & 3.034 & 41.75 & 41.41 & 45.63 & 3.716 & 0.30 & 0.68 & 0.78 \\
\hline Proteobacteria' & $1.42(6.05)$ & $1.45(7.04)$ & 0.111 & $0.88^{\mathrm{b}}(0.93)$ & $0.84^{b}(0.64)$ & $2.58^{\mathrm{a}}(18.06)$ & 0.120 & 0.68 & $<0.01$ & 0.47 \\
\hline Spirochaetes & 3.10 & 3.20 & 0.859 & $2.45^{\mathrm{b}}$ & $0.76^{b}$ & $6.23^{\mathrm{a}}$ & 0.941 & 0.90 & $<0.01$ & 0.59 \\
\hline Fibrobacteres & 0.49 & 0.56 & 0.214 & $1.16^{\mathrm{a}}$ & $0.12^{b}$ & $0.30^{\mathrm{b}}$ & 0.254 & 0.82 & $<0.01$ & 0.94 \\
\hline Actinobacteria & 0.59 & 0.85 & 0.115 & 0.65 & 0.85 & 0.66 & 0.141 & 0.11 & 0.55 & 0.84 \\
\hline
\end{tabular}

${ }^{\mathrm{a}-\mathrm{b}}$ Means with different superscripts within a row of REN differ $(P<0.05)$

${ }^{1}$ Data were cubic root transformed to ensure normality of residuals. Value in parenthesis is the mean of untransformed data in each treatment

the RL fraction, the relative abundance of 17 genera (including Butyrivibrio 2, Prevotellaceae UCG-001, Rikenellaceae U29-B03, Rikenellaceae Blvii28, Lachnospiraceae UCG-008, Defluviitaleaceae UCG-011, Eubacterium nodatum group, Erysipelotrichaceae uncultured, Lachnospiraceae UCG-010, Howardella, Halomonas, Desulfobulbus, Comamonas, Suttonella, Neisseriaceae uncultured, Campylobacter, and Treponema 2) was higher $(P<0.01)$, while that of five genera (including Prevotella 1, Prevotellaceae UCG-003, Christensenellaceae R-7 group, Ruminococcaceae NK4A214 group, and Lachnospiraceae XPB1014 group) was lower $(P<0.01)$ in the $\mathrm{RE}$ fraction than in the RS and the RL fractions. Seven genera, including Bacteroidales S24-7 group_norank, Succiniclasticum, Ruminococcus 1, Saccharofermentans, Eubacterium ruminantium group, Lachnospiraceae probable genus 10, and Fibrobacter, had a greater relative abundance $(P<0.01)$ in the RS than in the RL and RE fractions. In contrast, the RL fraction had a higher $(P<0.01)$ relative abundance of Bacteroidales BS11 gut group_norank, Bacteroidales RF16 group_norank, Ruminococcus 2, Eubacterium coprostanoligenes group, and Pseudobutyrivibrio, but a lower $(P<0.01)$ relative abundance of Ruminococcaceae UCG-014, than the RS and the RE fractions.

\section{Effect on inferred functional pathways}

The top 20 predominant level 2 metabolic pathways were shown in Table 4 . No interaction $(P \geq 0.10)$ of PS with REN was detected for any of the pathways. However, the relative abundance of the predominant pathways was altered differently by dietary PS or REN. Among the pathways, 9 out of 20 were significantly influenced by PS. Compared with the SBM-based diets, the DDGS-based diets resulted in higher $(P \leq 0.02)$ relative abundance of the functions related to amino acid metabolism, lipid metabolism, and metabolism of terpenoids and polyketides, but lower $(P \leq 0.04)$ relative abundance of the functions related to nucleotide metabolism, replication and repair, translation, cell motility, folding, sorting and degradation, infectious disease (bacterial), and cell growth and death. In contrast, 16 out of the predominant 20 pathways were significantly different $(P<$ $0.05)$ among the three REN. Compared with the RS and $\mathrm{RL}$, the RE fraction had greater $(P<0.01)$ predominance of the pathways related to amino acid metabolism, lipid metabolism, xenobiotics biodegradation and metabolism, and metabolism of terpenoids and polyketides, but lower $(P<0.01)$ predominance of the function related to membrane transport, signal transduction, and cell motility. The pathways related to replication and repair, biosynthesis of other secondary metabolites, and endocrine system were higher $(P \leq 0.02)$ in the RS than in the RL and RE fractions. In contrast, a higher $(P<0.01)$ relative abundance of the pathways related to the metabolism of cofactors and vitamins, and a lower $(P<0.01)$ relative abundance of the pathway related to the metabolism of other amino acids were observed in the RL than in the RS or the RE fractions.

\section{Effect on total bacteria, sulfate-reducing bacteria, methanogens, fungi, and protozoa}

No interaction $(P \geq 0.16)$ between PS and REN was detected with respect to the absolute abundance of total bacteria, SRB, methanogens, fungi, or protozoa (Table 5). The DDGS-fed lambs had a smaller $(P=0.04)$ population of protozoa than those fed SBM, but the population of total bacteria, SRB, fungi, and methanogens were similar $(P \geq 0.41)$. The populations of total bacteria and methanogens were lower $(P<0.05)$ in the RL fraction than in the RS fraction, but higher $(P<0.05)$ than in the RE fraction. The RS had a larger $(P<0.05)$ fungal population than the RL and the RE fractions, but no difference $(P>0.05)$ in the fungal population was noted between the RL and the RE fractions. Compared with the RS and the RL fractions, the RE fraction had a larger $(P<0.05)$ SRB population but a smaller $(P<0.05)$ protozoal population. However, no difference $(P>0.05)$ was observed in the populations SRB or protozoa between the RS and the RL fractions. 
Table 3 Effect of replacing soybean meal with dried distillers grains with solubles on relative abundance of major ruminal bacterial genera (each with a relative abundance $\geq 0.5 \%$ in at least one treatment) in different ruminal ecological niches of growing Hu lambs

\begin{tabular}{|c|c|c|c|c|c|c|c|c|c|c|c|}
\hline \multirow[t]{2}{*}{ Phylum } & \multirow[t]{2}{*}{ Genus/other } & \multicolumn{3}{|c|}{ Protein source (PS) } & \multicolumn{4}{|c|}{ Ruminal ecological niche (REN) } & \multicolumn{3}{|c|}{$P$-value } \\
\hline & & SBM & DDGS & SEM & Solid & Liquid & Epithelial & SEM & PS & REN & $\begin{array}{l}\text { PS } \times \\
\text { REN }\end{array}$ \\
\hline \multirow[t]{13}{*}{ Bacteroidetes } & Prevotella 1 & 19.19 & 19.50 & 2.779 & $25.01^{a}$ & $28.89^{a}$ & $3.94^{b}$ & 3.404 & 0.93 & $\begin{array}{l}< \\
0.01\end{array}$ & 0.83 \\
\hline & Rikenellaceae RC9 gut group & 7.09 & 5.76 & 0.589 & 6.59 & 7.13 & 5.57 & 0.721 & 0.12 & 0.31 & 0.39 \\
\hline & Prevotellaceae UCG-001 & 4.48 & 4.36 & 0.923 & $1.82^{b}$ & $1.23^{b}$ & $10.20^{\mathrm{a}}$ & 1.131 & 0.93 & $\begin{array}{l}< \\
0.01\end{array}$ & 0.93 \\
\hline & Bacteroidales S24-7 group & 3.66 & 3.06 & 0.549 & $6.56^{\mathrm{a}}$ & $2.42^{\mathrm{b}}$ & $1.09^{b}$ & 0.672 & 0.45 & $\begin{array}{l}< \\
0.01\end{array}$ & 0.25 \\
\hline & Bacteroidales BS11 gut group & 2.81 & 2.61 & 0.655 & $2.18^{b}$ & $4.23^{\mathrm{a}}$ & $1.72^{\mathrm{b}}$ & 0.765 & 0.80 & 0.03 & 0.88 \\
\hline & Prevotellaceae Unclassified ${ }^{1}$ & $\begin{array}{l}1.00 \\
(3.79)\end{array}$ & $\begin{array}{l}0.63 \\
(0.36)\end{array}$ & 0.165 & $0.83(1.54)$ & $0.87(3.97)$ & $0.74(0.72)$ & 0.195 & 0.08 & 0.86 & 0.72 \\
\hline & Prevotellaceae UCG-003 & 1.73 & 1.13 & 0.347 & $1.61^{\mathrm{a}}$ & $2.41^{\mathrm{a}}$ & $0.28^{b}$ & 0.404 & 0.16 & $\begin{array}{l}< \\
0.01\end{array}$ & 0.80 \\
\hline & Bacteroidetes Unclassified ${ }^{1}$ & $\begin{array}{l}0.87 \\
(1.24)\end{array}$ & $\begin{array}{l}0.69 \\
(0.48)\end{array}$ & 0.069 & $\begin{array}{l}1.01^{\mathrm{a}} \\
(1.39)\end{array}$ & $\begin{array}{l}0.89^{\mathrm{a}} \\
(1.07)\end{array}$ & $\begin{array}{l}0.43^{b} \\
(0.11)\end{array}$ & 0.085 & 0.08 & $\begin{array}{l}< \\
0.01\end{array}$ & 0.96 \\
\hline & Prevotellaceae NK3B31 group & 0.51 & 1.33 & 0.551 & 2.29 & 0.39 & 0.09 & 0.675 & 0.30 & 0.06 & 0.62 \\
\hline & Bacteroidetes VC2.1 Bac22 & 0.49 & 0.31 & 0.136 & $0.72^{\mathrm{a}}$ & $0.38^{\mathrm{ab}}$ & $0.10^{\mathrm{b}}$ & 0.150 & 0.18 & $\begin{array}{l}< \\
0.01\end{array}$ & 0.41 \\
\hline & Bacteroidales RF16 group & 0.71 & 0.62 & 0.212 & $0.12^{\mathrm{b}}$ & $1.64^{\mathrm{a}}$ & $0.25^{b}$ & 0.212 & 0.66 & $\begin{array}{l}< \\
0.01\end{array}$ & 0.97 \\
\hline & Rikenellaceae U29-B03 ${ }^{1}$ & $\begin{array}{l}0.53 \\
(0.25)\end{array}$ & $\begin{array}{l}0.60 \\
(0.55)\end{array}$ & 0.062 & $\begin{array}{l}0.46^{\mathrm{b}} \\
(0.12)\end{array}$ & $\begin{array}{l}0.38^{b} \\
(0.08)\end{array}$ & $\begin{array}{l}0.84^{\mathrm{a}} \\
(1.00)\end{array}$ & 0.077 & 0.43 & $\begin{array}{l}< \\
0.01\end{array}$ & 0.33 \\
\hline & Rikenellaceae Blvii28² & & & & ND & ND & 1.402 & & & & \\
\hline \multirow[t]{15}{*}{ Firmicutes } & Christensenellaceae R-7 group ${ }^{1}$ & $\begin{array}{l}1.86 \\
(7.47)\end{array}$ & $\begin{array}{l}2.01 \\
(8.18)\end{array}$ & 0.111 & $\begin{array}{l}1.99^{\mathrm{ab}} \\
(8.25)\end{array}$ & $\begin{array}{l}2.21^{\mathrm{a}} \\
(11.79)\end{array}$ & $\begin{array}{l}1.59^{b} \\
(3.43)\end{array}$ & 0.136 & 0.36 & 0.01 & 0.76 \\
\hline & Butyrivibrio $2^{1}$ & $\begin{array}{l}1.39 \\
(5.95)\end{array}$ & $\begin{array}{l}1.24 \\
(5.18)\end{array}$ & 0.146 & $\begin{array}{l}0.76^{b} \\
(0.57)\end{array}$ & $\begin{array}{l}0.79^{b} \\
(0.61)\end{array}$ & $\begin{array}{l}2.40^{\mathrm{a}} \\
(15.51)\end{array}$ & 0.156 & 0.16 & $\begin{array}{l}< \\
0.01\end{array}$ & 0.65 \\
\hline & $\begin{array}{l}\text { Ruminococcaceae NK4A214 } \\
\text { group }\end{array}$ & 3.63 & 4.38 & 0.461 & $4.66^{\mathrm{a}}$ & $5.95^{\mathrm{a}}$ & $1.40^{\mathrm{b}}$ & 0.564 & 0.25 & $\begin{array}{l}< \\
0.01\end{array}$ & 0.39 \\
\hline & Succiniclasticum & 1.99 & 1.39 & 0.270 & $2.60^{\mathrm{a}}$ & $0.99^{b}$ & $1.48^{\mathrm{b}}$ & 0.330 & 0.13 & $\begin{array}{l}< \\
0.01\end{array}$ & 0.92 \\
\hline & Ruminococcus 1 & 0.92 & 0.94 & 0.201 & $2.04^{\mathrm{a}}$ & $0.58^{\mathrm{b}}$ & $0.17^{\mathrm{b}}$ & 0.245 & 0.95 & $\begin{array}{l}< \\
0.01\end{array}$ & 0.84 \\
\hline & Lachnospiraceae NK3A20 group & 1.70 & 2.26 & 0.308 & 2.33 & 2.00 & 1.61 & 0.375 & 0.20 & 0.40 & 0.97 \\
\hline & Saccharofermentans & 0.82 & 1.03 & 0.112 & $1.88^{\mathrm{a}}$ & $0.75^{\mathrm{b}}$ & $0.16^{c}$ & 0.137 & 0.18 & $\begin{array}{l}< \\
0.01\end{array}$ & 0.38 \\
\hline & Ruminococcus 2 & 1.33 & 1.47 & 0.484 & $0.98^{\mathrm{b}}$ & $3.11^{\mathrm{a}}$ & $0.11^{\mathrm{b}}$ & 0.559 & 0.80 & $\begin{array}{l}< \\
0.01\end{array}$ & 0.76 \\
\hline & $\begin{array}{l}\text { Eubacterium coprostanoligenes } \\
\text { group }\end{array}$ & 0.69 & 0.58 & 0.068 & $0.74^{\mathrm{b}}$ & $1.08^{\mathrm{a}}$ & $0.10^{c}$ & 0.082 & 0.25 & $\begin{array}{l}< \\
0.01\end{array}$ & 0.13 \\
\hline & Ruminococcaceae UCG-014 & 0.91 & 1.08 & 0.112 & $1.10^{\mathrm{a}}$ & $0.55^{\mathrm{b}}$ & $1.33^{\mathrm{a}}$ & 0.138 & 0.28 & $\begin{array}{l}< \\
0.01\end{array}$ & 0.37 \\
\hline & Eubacterium ruminantium group & 0.32 & 0.44 & 0.102 & $0.87^{\mathrm{a}}$ & $0.19^{b}$ & $0.07^{\mathrm{b}}$ & 0.113 & 0.24 & $\begin{array}{l}< \\
0.01\end{array}$ & 0.48 \\
\hline & $\begin{array}{l}\text { Lachnospiraceae XPB1014 } \\
\text { group }^{1}\end{array}$ & $\begin{array}{l}0.64 \\
(0.60)\end{array}$ & $\begin{array}{l}0.81 \\
(1.08)\end{array}$ & 0.085 & $\begin{array}{l}0.93^{\mathrm{a}} \\
(1.35)\end{array}$ & $\begin{array}{l}0.86^{\mathrm{a}} \\
(1.10)\end{array}$ & $\begin{array}{l}0.38^{b} \\
(0.07)\end{array}$ & 0.104 & 0.18 & $\begin{array}{l}< \\
0.01\end{array}$ & 0.60 \\
\hline & Ruminococcaceae UCG-005 & 0.70 & 0.23 & 0.078 & 0.36 & 0.41 & 0.63 & 0.096 & $\begin{array}{l}< \\
0.01\end{array}$ & 0.11 & 0.95 \\
\hline & $\begin{array}{l}\text { Lachnospiraceae probable genus } \\
10\end{array}$ & 0.24 & 0.34 & 0.093 & $0.694^{\mathrm{a}}$ & $0.135^{b}$ & $0.046^{b}$ & 0.113 & 0.47 & $\begin{array}{l}< \\
0.01\end{array}$ & 0.81 \\
\hline & Ruminococcaceae UCG-010 & 0.46 & 0.41 & 0.063 & $0.58^{\mathrm{a}}$ & $0.45^{\mathrm{ab}}$ & $0.29^{b}$ & 0.078 & 0.60 & 0.048 & 0.19 \\
\hline
\end{tabular}


Table 3 Effect of replacing soybean meal with dried distillers grains with solubles on relative abundance of major ruminal bacterial genera (each with a relative abundance $\geq 0.5 \%$ in at least one treatment) in different ruminal ecological niches of growing Hu lambs (Continued)

\begin{tabular}{|c|c|c|c|c|c|c|c|c|c|c|c|}
\hline \multirow[t]{2}{*}{ Phylum } & \multirow[t]{2}{*}{ Genus/other } & \multicolumn{3}{|c|}{ Protein source (PS) } & \multicolumn{4}{|c|}{ Ruminal ecological niche (REN) } & \multicolumn{3}{|c|}{$P$-value } \\
\hline & & SBM & DDGS & SEM & Solid & Liquid & Epithelial & SEM & PS & REN & $\begin{array}{l}\text { PS } \times \\
\text { REN }\end{array}$ \\
\hline & Lachnospiraceae uncultured & 0.30 & 0.38 & 0.108 & $0.63^{\mathrm{a}}$ & $0.32^{\mathrm{ab}}$ & $0.07^{\mathrm{b}}$ & 0.125 & 0.56 & $\begin{array}{l}< \\
0.01\end{array}$ & 0.79 \\
\hline & Family XIII AD3011 group & 0.51 & 0.50 & 0.052 & 0.51 & 0.42 & 0.58 & 0.063 & 0.86 & 0.23 & 0.98 \\
\hline & Pseudobutyrivibrio $^{3}$ & 0.44 & 1.06 & 0.135 & $0.56^{\mathrm{b}}$ & $1.62^{\mathrm{a}}$ & $0.06^{\mathrm{b}}$ & 0.165 & $\begin{array}{l}< \\
0.01\end{array}$ & $\begin{array}{l}< \\
0.01\end{array}$ & $<0.01$ \\
\hline & Lachnospiraceae Unclassified' & $\begin{array}{l}0.73 \\
(0.45)\end{array}$ & $\begin{array}{l}0.83 \\
(0.81)\end{array}$ & 0.097 & $0.82(0.70)$ & $0.74(0.63)$ & $0.77(0.56)$ & 0.105 & 0.23 & 0.69 & 0.39 \\
\hline & Lachnospiraceae AC2044 group' & $\begin{array}{l}0.48 \\
(0.18)\end{array}$ & $\begin{array}{l}0.54 \\
(0.55)\end{array}$ & $\begin{array}{l}0.074 \\
0.199\end{array}$ & $\begin{array}{l}0.73^{\mathrm{a}} \\
(0.82)\end{array}$ & $\begin{array}{l}0.44^{\mathrm{ab}} \\
(0.20)\end{array}$ & $\begin{array}{l}0.37^{b} \\
(0.07)\end{array}$ & 0.090 & 0.54 & 0.02 & 0.92 \\
\hline & Anaerovibrio & 0.28 & 0.25 & 0.069 & $0.25^{\mathrm{ab}}$ & $0.51^{\mathrm{a}}$ & $0.03^{\mathrm{b}}$ & 0.084 & 0.75 & $\begin{array}{l}< \\
0.01\end{array}$ & 0.97 \\
\hline & Anaerovorax & 0.26 & 0.45 & 0.102 & $0.27^{\mathrm{ab}}$ & $0.13^{b}$ & $0.65^{\mathrm{a}}$ & 0.102 & 0.20 & 0.02 & 0.24 \\
\hline & Lachnospiraceae UCG-008 & $\begin{array}{l}0.85 \\
(1.34)\end{array}$ & $\begin{array}{l}0.73 \\
(0.65)\end{array}$ & 0.049 & $\begin{array}{l}0.58^{b} \\
(0.21)\end{array}$ & $\begin{array}{l}0.50^{b} \\
(0.13)\end{array}$ & $\begin{array}{l}1.29^{\mathrm{a}} \\
(2.65)\end{array}$ & 0.060 & 0.11 & $\begin{array}{l}< \\
0.01\end{array}$ & 0.32 \\
\hline & Defluviitaleaceae UCG-011 & 0.30 & 0.25 & 0.089 & $0.16^{\mathrm{b}}$ & $0.07^{b}$ & $0.59^{\mathrm{a}}$ & 0.109 & 0.69 & $\begin{array}{l}< \\
0.01\end{array}$ & 0.97 \\
\hline & Eubacterium nodatum group ${ }^{1}$ & $\begin{array}{l}0.80 \\
(1.10)\end{array}$ & $\begin{array}{l}0.88 \\
(1.45)\end{array}$ & 0.037 & $\begin{array}{l}0.57^{b} \\
(0.20)\end{array}$ & $\begin{array}{l}0.46^{b} \\
(0.11)\end{array}$ & $\begin{array}{l}1.49^{\mathrm{a}} \\
(3.52)\end{array}$ & 0.045 & 0.16 & $\begin{array}{l}< \\
0.01\end{array}$ & 0.69 \\
\hline & Erysipelotrichaceae uncultured & 0.38 & 0.42 & 0.068 & $0.20^{\mathrm{b}}$ & $0.11^{\mathrm{b}}$ & $0.89^{\mathrm{a}}$ & 0.077 & 0.61 & $\begin{array}{l}< \\
0.01\end{array}$ & 0.98 \\
\hline & Lachnospiraceae UCG-010 & $\begin{array}{l}0.37 \\
(0.21)\end{array}$ & $\begin{array}{l}0.36 \\
(0.16)\end{array}$ & 0.034 & $\begin{array}{l}0.15^{\mathrm{b}} \\
(0.009)\end{array}$ & $\begin{array}{l}0.16^{\mathrm{b}} \\
(0.008)\end{array}$ & $\begin{array}{l}0.78^{a} \\
(0.545)\end{array}$ & 0.042 & 0.86 & $\begin{array}{l}< \\
0.01\end{array}$ & 0.32 \\
\hline & Howardella ${ }^{1}$ & $\begin{array}{l}0.52 \\
(0.43)\end{array}$ & $\begin{array}{l}0.58 \\
(0.61)\end{array}$ & 0.031 & $\begin{array}{l}0.14^{c} \\
(0.004)\end{array}$ & $\begin{array}{l}0.40^{\mathrm{b}} \\
(0.071)\end{array}$ & $\begin{array}{l}1.11^{\mathrm{a}} \\
(1.49)\end{array}$ & 0.038 & 0.14 & $\begin{array}{l}< \\
0.01\end{array}$ & 0.67 \\
\hline \multirow[t]{6}{*}{ Proteobacteria } & Halomonas $^{1}$ & $\begin{array}{l}0.63 \\
(0.56)\end{array}$ & $\begin{array}{l}0.54 \\
(0.50)\end{array}$ & 0.038 & $\begin{array}{l}0.26^{b} \\
(0.03)\end{array}$ & $\begin{array}{l}0.40^{b} \\
(0.07)\end{array}$ & $\begin{array}{l}1.10^{\mathrm{a}} \\
(1.49)\end{array}$ & 0.046 & 0.10 & $\begin{array}{l}< \\
0.01\end{array}$ & 0.26 \\
\hline & Desulfobulbus ${ }^{1}$ & $\begin{array}{l}0.64 \\
(1.47)\end{array}$ & $\begin{array}{l}0.74 \\
(2.03)\end{array}$ & 0.056 & $\begin{array}{l}0.13^{b} \\
(0.004)\end{array}$ & $\begin{array}{l}0.28^{b} \\
(0.031)\end{array}$ & $\begin{array}{l}1.66^{\mathrm{a}} \\
(5.22)\end{array}$ & 0.069 & 0.24 & $\begin{array}{l}< \\
0.01\end{array}$ & 0.39 \\
\hline & Comamonas $^{1}$ & $\begin{array}{l}0.35 \\
(0.44)\end{array}$ & $\begin{array}{l}0.36 \\
(0.45)\end{array}$ & 0.057 & $\begin{array}{l}0.03^{b} \\
(0.001)\end{array}$ & $\begin{array}{l}0.07^{\mathrm{b}} \\
(0.002)\end{array}$ & $\begin{array}{l}0.96^{\mathrm{a}} \\
(1.34)\end{array}$ & 0.098 & 0.91 & $\begin{array}{l}< \\
0.01\end{array}$ & 0.97 \\
\hline & Suttonella $a^{1,3}$ & $\begin{array}{l}0.42 \\
(0.31)\end{array}$ & $\begin{array}{l}0.28 \\
(0.08)\end{array}$ & 0.028 & $\begin{array}{l}0.06^{c} \\
(0.002)\end{array}$ & $\begin{array}{l}0.22^{b} \\
(0.016)\end{array}$ & $\begin{array}{l}0.76^{\mathrm{a}} \\
(0.562)\end{array}$ & 0.034 & $\begin{array}{l}< \\
0.01\end{array}$ & $\begin{array}{l}< \\
0.01\end{array}$ & $<0.01$ \\
\hline & Neisseriaceae uncultured ${ }^{2}$ & & & & ND & ND & 2.66 & & & & \\
\hline & Campylobacter ${ }^{2}$ & & & & ND & ND & 5.62 & & & & \\
\hline Spirochaetes & Treponema 2 & 2.90 & 3.05 & 0.851 & $2.44^{\mathrm{b}}$ & $0.74^{b}$ & $5.74^{\mathrm{a}}$ & 0.932 & 0.84 & $\begin{array}{l}< \\
0.01\end{array}$ & 0.51 \\
\hline Fibrobacteres & Fibrobacter & 0.49 & 0.55 & 0.213 & $1.15^{\mathrm{a}}$ & $0.11^{b}$ & $0.30^{\mathrm{b}}$ & 0.235 & 0.83 & $\begin{array}{l}< \\
0.01\end{array}$ & 0.94 \\
\hline
\end{tabular}

\footnotetext{
${ }^{\mathrm{a}-\mathrm{c}}$ Means with different superscripts within a row of REN differ $(P<0.05)$

${ }^{1}$ Data were cubic root transformed to ensure normality of residuals. Value in parenthesis is the mean of untransformed data in each treatment ${ }^{2}$ Genus/other can not be detected the rumen solid and liquid fraction, and only the difference of relative abundance of bacterial genera from rumen epithelium (RE) between SBM and DDGS (SBM-RE and DDGS-RE) was analyzed with SAS. Relative abundance (least square mean) of Blvii28, Neisseriaceae uncultured, and Campylobacter (\%) for SBM-RE and DDGS-RE were 1.52 and $1.28(P=0.68) ; 2.52$ and $2.80(P=0.88) ; 4.62$ and $6.62(P=0.50)$, respectively

${ }^{3}$ Relative abundance (least square mean) of Pseudobutyrivibrio (\%) was $0.52^{\mathrm{b}}, 0.74^{\mathrm{b}}, 0.07^{\mathrm{b}}, 0.61^{\mathrm{b}}, 2.52^{\mathrm{a}}$ and $0.06^{\mathrm{b}}$ for SBM-RS, SBM-RL, SBM-RE, DDGS-RS, DDGS-RL, and DDGS-RE, respectively, and that of Suttonella was $0.001^{\mathrm{b}}, 0.023^{\mathrm{b}}, 0.899^{\mathrm{a}}, 0.002^{\mathrm{b}}, 0.009^{\mathrm{b}}$, and $0.225^{\mathrm{b}}$ for SBM-RS, SBM-RL, SBM-RE, DDGS-RS, DDGS-RL, and DDGS-RE, respectively. DDGS: dried distillers grains with solubles, SBM: soybean meal, RE: rumen epithelium, RS: rumen solid, RL: rumen liquid
} 
Table 4 Effect of replacing soybean meal with dried distillers grains with solubles on relative abundance of predominant predicted gene pathways in the different ruminal ecological niches of growing Hu lambs

\begin{tabular}{|c|c|c|c|c|c|c|c|c|c|c|}
\hline \multirow[t]{2}{*}{ Metabolism } & \multicolumn{3}{|c|}{ Protein source (PS) } & \multicolumn{4}{|c|}{ Ruminal ecological niche (REN) } & \multicolumn{3}{|c|}{$P$-value } \\
\hline & SBM & DDGS & SEM & Solid & Liquid & Epithelial & SEM & PS & REN & $P S \times R E N$ \\
\hline Carbohydrate metabolism & 10.68 & 10.83 & 0.058 & 10.79 & 10.63 & 10.85 & 0.070 & 0.08 & 0.096 & 0.74 \\
\hline Amino acid metabolism & 6.78 & 7.02 & 0.066 & $6.72^{\mathrm{b}}$ & $6.84^{b}$ & $7.14^{\mathrm{a}}$ & 0.080 & 0.02 & $<0.01$ & 0.99 \\
\hline Energy metabolism & 4.48 & 4.45 & 0.029 & 4.47 & 4.52 & 4.41 & 0.036 & 0.51 & 0.12 & 0.81 \\
\hline Nucleotide metabolism & 4.74 & 4.57 & 0.051 & $4.79^{\mathrm{a}}$ & $4.48^{\mathrm{b}}$ & $4.69^{a b}$ & 0.063 & 0.02 & $<0.01$ & 0.86 \\
\hline Replication and repair & 4.31 & 4.09 & 0.056 & $4.42^{\mathrm{a}}$ & $4.07^{b}$ & $4.11^{\mathrm{b}}$ & 0.069 & $<0.01$ & $<0.01$ & 0.96 \\
\hline Metabolism of cofactors and vitamins & 3.38 & 3.28 & 0.036 & $3.25^{\mathrm{b}}$ & $3.52^{\mathrm{a}}$ & $3.22^{\mathrm{b}}$ & 0.044 & 0.07 & $<0.01$ & 0.66 \\
\hline Membrane transport & 2.71 & 2.63 & 0.050 & $2.81^{\mathrm{a}}$ & $2.75^{\mathrm{a}}$ & $2.46^{\mathrm{b}}$ & 0.062 & 0.23 & $<0.01$ & 0.10 \\
\hline Signal transduction & 2.49 & 2.36 & 0.056 & $2.51^{\mathrm{a}}$ & $2.63^{\mathrm{a}}$ & $2.13^{b}$ & 0.068 & 0.11 & $<0.01$ & 0.18 \\
\hline Translation & 2.68 & 2.52 & 0.051 & $2.73^{\mathrm{a}}$ & $2.49^{\mathrm{b}}$ & $2.58^{\mathrm{ab}}$ & 0.061 & 0.03 & 0.02 & 0.82 \\
\hline Cell motility & 1.85 & 1.62 & 0.070 & $1.81^{\mathrm{a}}$ & $2.11^{\mathrm{a}}$ & $1.29^{\mathrm{b}}$ & 0.085 & 0.03 & $<0.01$ & 0.40 \\
\hline Lipid metabolism & 1.62 & 1.74 & 0.032 & $1.61^{\mathrm{b}}$ & $1.56^{\mathrm{b}}$ & $1.86^{\mathrm{a}}$ & 0.039 & $<0.01$ & $<0.01$ & 0.75 \\
\hline Folding, sorting and degradation & 1.56 & 1.50 & 0.014 & $1.58^{\mathrm{a}}$ & $1.53^{\mathrm{ab}}$ & $1.49^{\mathrm{b}}$ & 0.018 & $<0.01$ & $<0.01$ & 0.97 \\
\hline Metabolism of other amino acids & 1.36 & 1.39 & 0.019 & $1.40^{\mathrm{a}}$ & $1.31^{\mathrm{b}}$ & $1.41^{\mathrm{a}}$ & 0.023 & 0.32 & $<0.01$ & 0.67 \\
\hline Glycan biosynthesis and metabolism & 1.21 & 1.20 & 0.021 & 1.17 & 1.22 & 1.21 & 0.026 & 0.73 & 0.27 & 0.94 \\
\hline Biosynthesis of other secondary metabolites & 1.08 & 1.07 & 0.008 & $1.11^{\mathrm{a}}$ & $1.04^{\mathrm{b}}$ & $1.07^{b}$ & 0.010 & 0.24 & $<0.01$ & 0.87 \\
\hline Xenobiotics biodegradation and metabolism & 1.02 & 1.14 & 0.033 & $1.06^{\mathrm{b}}$ & $0.90^{c}$ & $1.28^{\mathrm{a}}$ & 0.040 & 0.01 & $<0.01$ & 0.95 \\
\hline Metabolism of terpenoids and polyketides & 0.87 & 0.93 & 0.025 & $0.85^{\mathrm{b}}$ & $0.84^{\mathrm{b}}$ & $1.01^{\mathrm{a}}$ & 0.027 & $<0.01$ & $<0.01$ & 0.21 \\
\hline Infectious diseases: Bacterial & 1.56 & 1.50 & 0.014 & $1.58^{\mathrm{a}}$ & $1.53^{\mathrm{ab}}$ & $1.49^{\mathrm{b}}$ & 0.014 & $<0.01$ & $<0.01$ & 0.97 \\
\hline Endocrine system & 0.85 & 0.82 & 0.017 & $0.88^{\mathrm{a}}$ & $0.81^{\mathrm{b}}$ & $0.81^{\mathrm{b}}$ & 0.021 & 0.17 & 0.02 & 0.24 \\
\hline Cell growth and death & 0.64 & 0.61 & 0.009 & 0.63 & 0.63 & 0.61 & 0.011 & 0.04 & 0.25 & 0.31 \\
\hline
\end{tabular}

${ }^{a-b}$ Means with different superscripts within a row differ $(P<0.05)$

\section{Discussion}

\section{Effects of dietary protein source on rumen microbiota structure and function}

Diet is one of the main factors influencing the structure and function of rumen microbiota [2, 3]. In our previous feeding trial, replacing SBM in an isonitrogenous lamb diet with 20\% DDGS increased dietary EE, NDF and ADF contents [5]. Therefore, we expected shifts of rumen bacterial community and structure. However, high throughput sequencing results (Tables 1, 2 and 3) revealed very limited influence of dietary PS on the bacterial community structure. These results are similar to a previous study that reported no or little difference in microbiota diversity and relative abundance of most bacteria in the rumen of crossbred steers fed 19.5\% DDGS replacing corn bran [11] or dairy cows fed 20\% DDGS replacing SBM [13]. In contrast, Callaway et al. [9] and Ramirez-Ramirez et al. [12] found drastic changes in rumen bacterial community structure when DDGS were added at $30 \%$ and $50 \%$ of the diet [dry matter (DM)].

Table 5 Effect of replacing soybean meal with dried distillers grains with solubles on the absolute abundance total bacteria, sulfurreducing bacteria, fungi, protozoa, and methanogens ( $\log _{10}$ copies of the target genes/g sample) in different ruminal ecological niches of growing Hu lambs

\begin{tabular}{|c|c|c|c|c|c|c|c|c|c|c|}
\hline \multirow[t]{2}{*}{ Item } & \multicolumn{3}{|c|}{ Protein source (PS) } & \multicolumn{4}{|c|}{ Ruminal ecological niche (REN) } & \multicolumn{3}{|c|}{$P$-value } \\
\hline & SBM & DDGS & SEM & Solid & Liquid & Epithelial & SEM & PS & REN & $P S \times R E N$ \\
\hline Total bacteria & 12.32 & 12.31 & 0.066 & $12.81^{a}$ & $12.31^{\mathrm{b}}$ & $11.83^{c}$ & 0.073 & 0.76 & $<0.01$ & 0.44 \\
\hline Sulfur-reducing bacteria & 8.50 & 8.62 & 0.138 & $8.24^{b}$ & $8.03^{b}$ & $9.42^{\mathrm{a}}$ & 0.154 & 0.41 & $<0.01$ & 0.89 \\
\hline Fungi & 9.61 & 9.55 & 0.104 & $11.52^{\mathrm{a}}$ & $8.65^{b}$ & $8.56^{b}$ & 0.128 & 0.69 & $<0.01$ & 0.26 \\
\hline Protozoa & 8.93 & 8.52 & 0.156 & $9.46^{\mathrm{a}}$ & $9.16^{\mathrm{a}}$ & $7.55^{\mathrm{b}}$ & 0.183 & 0.04 & $<0.01$ & 0.93 \\
\hline Methanogen & 10.01 & 10.03 & 0.058 & $10.41^{a}$ & $10.15^{b}$ & $9.50^{c}$ & 0.071 & 0.79 & $<0.01$ & 0.16 \\
\hline
\end{tabular}

\footnotetext{
${ }^{\mathrm{a}-\mathrm{c}}$ Means with different superscripts within a row differ $(P<0.05)$
} 
The disparity in bacterial community structure among different studies may be due to differences in DDGS feeding level and the chemical composition of the diets [13]. Additionally, the difference of ruminant species, duration of experimental period, sequencing and data analysis methods may also contribute to the discrepancy in the bacterial community among studies.

Although the bacterial community structure in the rumen did not differ much between the two different protein sources, the metabolic pathways predicted from the 16S rRNA gene sequences varied greatly (Table 4). These results demonstrated that the substitution of SBM with DDGS had a greater impact to the rumen microbial function than to the bacterial composition. It is also suggested that small changes in the rumen bacterial community may lead to greater variations in metabolic pathways. Among the predicted metabolic pathways, the increase of lipid metabolism pathway in the DDGS treatment group might be related to the higher EE content in the diet. The changes in other metabolic pathways, such as amino acid metabolism, metabolism of terpenoids and polyketides, nucleotide metabolism, replication and repair, translation, cell motility, might also be related to changes in dietary composition, but the specific mechanism is still unclear. The application of multiple metaomics, such as metagenomics, metatranscriptomics, metaproteomics, and metabolomics [27] in future studies may help further explain the effect of different protein sources on rumen metabolism.

Feed digestion and fermentation are the concerted functions of a variety of microorganisms including bacteria, fungi, protozoa, archaea and phages [27]. Therefore, the change of rumen fermentation parameters found in our previous feeding trial [5] might be closely associated with shifts of members of the rumen microbiota besides bacteria. This study showed that the populations of rumen total bacteria, fungi, SRB, and methanogens were not influenced by the dietary protein sources, but the population of protozoa was reduced by the DDGS substitution for SBM (Table 5). Protozoa can account for up to $50 \%$ of the rumen biomass and play an important role in the degradation of dietary fiber and protein [38]. Moreover, protozoa are also known to increase ammonia production [39]. In a previous metaanalysis, it has been reported that the elimination of protozoa from the rumen significantly decreased VFA and ammonia concentration [40]. Therefore, the decreased protozoal population might have primarily contributed to the reduced VFA and ammonia concentration in the DDGS group [5]. These findings indicate that, in addition to bacteria, the structure and function of other microbes such as protozoa, fungi, methanogens, and phages should also be studied in nutritional research.

\section{Effects of ruminal ecological niche on microbiota structure and function}

Consistent with previous studies using PCR-DGGE [17] or high-throughput sequencing $[15,18,26], \mathrm{RS}$ and RL shared more bacteria when compared to RE. In addition, the predicted functions and the qPCR results of the microbial groups further demonstrated that there were great differences in the structure and function of the microbiota occupying different REN. This corroborates the niche partitioning of the rumen microbiota.

\section{Differences in microbiota between rumen solid and liquid fractions}

The Shannon diversity index in the RS is higher than in the RL (Table 1), which agrees with the findings of previous studies $[15,18]$. The bacterial richness and taxonomic composition in the RS and RL were similar, suggesting continuous exchange between these two fractions [15]. However, some taxa had different relative abundance in these two niches, a finding consistent with other reports $[15,18,26]$ and likely reflecting specialized niches related to digestion of soluble components vs. dietary fiber [23].

Rumen bacteria found in the RS fraction are mainly responsible for the initial and secondary degradation of feed and play an important role in fiber digestion, while the bacteria in the RL fraction are mainly involved in the fermentation of soluble nutrients and metabolic end products of feed digestion [15, 41]. Ruminococcus and Fibrobacter are the two main known fibrolytic bacterial genera in the rumen [42]. We indeed found a higher predominance of Ruminococcus 1 and Fibrobacter spp. in the RS than in the RL fractions of the growing lambs (Table 3). Some other genera, such as Bacteroidales S24-7 group, Succiniclasticum, Saccharofermentans, Eubacterium ruminantium group, and Lachnospiraceae probable genus 10 , were also more predominant in the RS than in the RL, which indicates that these bacteria may play an important role in the initial and secondary degradation of feed. In contrast, some other bacterial genera, such as Bacteroidales BS11 gut group, Bacteroidales RF16 group, Ruminococcus 2, Eubacterium coprostanoligenes group, and Pseudobytyrivibrio showed the opposite trend, which indicates that these bacteria probably are mainly involved in the catabolism of soluble nutrients. Moreover, the results of functional analysis further demonstrated that there were great differences in multiple bacterial metabolic pathways between the RS and the RL.

It should be noted that bacteria with similar relative abundance in the RS and the RL fraction may differ in their absolute abundance. In the present study, the population of total bacteria in the RS is much higher than that in the RL (Table 5), indicating that the population of bacteria might be greater in the RS than in the 
RL. In addition to bacteria, other rumen microorganisms such as protozoa, fungi and methanogens also play an important role in feed digestion [27]. However, recent studies mainly focused on the diversity and function of rumen bacteria, ignoring the structure and function of other microbial microbes [27]. The rumen fungi can account for up to $20 \%$ of the microbial biomass and play an important role in ruminal fiber degradation [43]. Methanogens are the main hydrogen utilizing microorganisms in the rumen, and hydrogen produced by hydrogen-producing bacteria such as cellulolytic bacteria and fungi are used by methanogens to reduce $\mathrm{CO}_{2}$ to methane [44]. Therefore, it is not surprising that the population of fungi and methanogens is higher in the RS than in the RL. In contrast, the similar population of protozoa between the RS and the RL in the rumen is probably due to the ability of protozoa to freely attach to and dissociate from feed particles [41]. Generally, the population of protozoa is more abundant than fungi in rumen [45]. In the present study, similar results was also found in the RL fraction. However, the population of fungi in RS fraction is much higher than protozoa. Therefore, the disparity with previous reports in the population of rumen fungi and protozoa may be due to differences in REN.

\section{Differences in microbiota between epithelial and the solid or the liquid fractions}

In line with previous studies $[15,18,26]$, the RE bacterial diversity was lower than that in the RS or the RL fraction (Table 2), and the bacterial composition was greatly distinct from that of the RS or the RL (Table 3). At the phylum level, contrary to the finding in the RS or the RL, Firmicutes was the first while Bacteroidetes the second largest phyla in the RE microbiota. It is worth noting that Proteobacteria was the third largest phylum (with a relative abundance of $18.06 \%$ ) of the RE microbiota, which is consistent with previous studies on dairy cattle [19, 24]. Members of Proteobacteria are mostly facultative anaerobes [46]. Therefore, the higher predominance of Proteobacteria on RE could be explained by the trace amounts of oxygen diffused through the rumen tissue [47]. Interestingly, all the dominant bacterial genera classified to Proteobacteria in the epithelial microbiota, such as Campylobacter, Desulfobulbus, Neisseriaceae uncultured, Comamonas, and Halomonas were barely detected in the RS or the RL. In addition to bacteria of Proteobacteria, some bacterial genera assigned to other phyla were predominant on the RE (e.g., Prevotellaceae UCG-001, Butyrivibrio 2, and Treponema 2), and again, they are virtually undetected or only detected at much lower relative abundance in the RS and or the RL. These results support the notion that the distinctive epithelial bacteria assigned to different phyla may have additional functions other than feed digestion [21].
Corroborating the finding of previous studies [26, 48, 49], Desulfobulbus, one genus of SRB [50], was the predominant SRB genus (5.22\%) in RE microbiota (Table 3). In line with the high-throughput sequencing result, the qPCR results also demonstrated that SRB were more predominant on RE than in the RS or the RL fraction. Previous studies have shown that sulfides produced by SRB can disrupt the gut epithelial tissues and induce DNA damage, and adversely affect host health [51, 52]. However, it was found that the relative abundance of Butyrivibrio 2 (15.51\%) on RE was about 3 times higher than that of Desulfobulbus. Members of Butyrivibrio (e.g., Butyrivibrio fibrisolvens) are important butyrate producing bacteria ubiquitous in the rumen [53]. In vitro cell culture experiments have shown that butyrate could regulate colonic proliferation and treat ulcerative colitis caused by sulfide [54]. Therefore, it is also reasonable to believe that butyrate, a metabolite of Butyrivibrio spp., may help repair the damage to the RE caused by the sulfide produced by SRB (e.g., Desulfobulbus spp.). Based on this, we speculate that there is a self-regulating mechanism between the epithelial microbes and the host that helps the homeostasis of RE function.

Rumen epithelial microbiota play an important role in digesting and recycling the keratinized distal cells of the epithelium [14]. In the present study, the enhanced pathway of amino acid metabolism and lipid metabolism in the RE microbiota compared with the RS and the RL microbiota (Table 4) are probably related with the special nutrient composition of the keratinized distal cells of the epithelium. One recent metatranscriptomic analysis of RE microbiota showed that many metabolic genes encoding enzymes involved in $\mathrm{N}$ metabolism such as glutamate dehydrogenase, glutamine synthase and glutamate synthase were highly expressed, which demonstrated the importance of RE bacteria in $\mathrm{N}$ metabolism [55]. Similarly, Mao et al. [19] also found enhanced amino acid metabolism in the RE microbiota compared with the microbiota of rumen content. In the present study, the qPCR results confirmed the existence of fungi, protozoa and methanogens in the epithelial microbiota. However, Mann et al. [55] detected fungi and methanogens but no protozoa on the RE in their metatranscriptomic analysis. The inconsistent results might be explained by the more sensitive detection by qPCR than by RNA-seq.

\section{Conclusions}

This study, combing high-throughput sequencing, functional prediction, and $\mathrm{qPCR}$, for the first time, revealed the difference of structure and function of rumen microbiota occupying different REN of growing $\mathrm{Hu}$ lambs in response to alterations in dietary PS. The results of the present study indicate that substitution of SBM with DDGS had greater impact to protozoa than to other 
microbes, and the microbiota structure and function in different REN are specific and niche-adapted. In order to have a better understanding of the complex rumen ecosystem, in addition to bacteria, the structure and function of other microbes such as protozoa, fungi, and methanogens should also be studied.

\section{Supplementary information}

Supplementary information accompanies this paper at https://doi.org/10. 1186/s40104-020-00499-2.

Additional file 1 : Table $\mathbf{S 1}$. Primers used for real-time PCR quantification of rumen target organisms. Figure S1. Box plots showing withingroup similarity and between-group dissimilarity of rumen microbiota based on Bray-Curtis dissimilarity in different ruminal ecological niches of growing Hu lambs in response to alterations in dietary protein sources. The different letters denote significant differences (Kruskal-Wallis tests, FDR-adjusted $\mathrm{q}<0.05)$. DDGS: dried distillers grains with solubles, SBM: soybean meal, RE: rumen epithelium, RS: rumen solid, RL: rumen liquid.

\section{Abbreviations}

ADF: Acid detergent fiber; ANOSIM: Analysis of similarity for multivariate; CP: Crude protein; DDGS: Dried distillers grains with solubles; DE: Digestible energy; DM: Dry matter; DNA: Deoxyribonucleic acid; dsrA: Dissimilatory sulfite reductase alpha subunit gene; EE: Ether extract; KEGG: Kyoto Encyclopedia of Genes and Genomes; mcrA: Methyl coenzyme-M reductase alpha subunit gene; N: Nitrogen; NDF: Neutral detergent fiber;

OTUs: Operational taxonomic units; PCoA: Principal coordinates analysis; PCRDGGE: Polymerase chain reaction-Denaturing gradient gel electrophoresis; PS: Protein source; GPCR: Real-time quantitative polymerase chain reaction; RE: Rumen epithelium; REN: Ruminal ecological niche; RL: Rumen liquid; RS: Rumen solid; SBM: Soybean meal; SRB: Sulfur-reducing bacteria; TMR: Total mixed ration; VFA: Volatile fatty acid

\section{Acknowledgements}

The authors thank all of the staff of the Shanghai Yonghui Sheep Industry Co., Ltd. for their assistance in animal care and sample collection.

\section{Authors' contributions}

JS and WZ conceived and designed the experiment. JS performed the experiment. JS and ZL analyzed the data. JS wrote the paper. ZL, ZY and WZ edited and reviewed the manuscript. All authors read and approved the final manuscript.

\section{Funding}

This work was partially supported by the National Natural Science Foundation of China (award No: 31402101), the "One Belt and One Road" Technical Cooperation Project of Jiangsu Province (award No: BZ2018055), and the Jiangsu Agriculture Science and Technology Innovation Fund (award No: CX(19)3023).

\section{Availability of data and materials}

All data generated or analyzed during this study are included in this published article [and its supplementary information files].

\section{Ethics approval and consent to participate}

The experimental procedures used in this study were approved by the Animal Care and Use Committee of Nanjing Agricultural University (protocol number: SYXK2017-0007)

\section{Consent for publication}

Not applicable.

\section{Competing interests}

The authors declare no competing interests.

\section{Author details}

'Laboratory of Gastrointestinal Microbiology, Jiangsu Key Laboratory of Gastrointestinal Nutrition and Animal Health, College of Animal Science and Technology, Nanjing Agricultural University, Nanjing 210095, China. ${ }^{2}$ National Center for International Research on Animal Gut Nutrition, Nanjing Agricultural University, Nanjing 210095, China. ${ }^{3}$ Department of Special Animal Nutrition and Feed Science, Institute of Special Animal and Plant Sciences, Chinese Academy of Agricultural Sciences, Changchun 130112, China. ${ }^{4}$ Department of Animal Sciences, The Ohio State University, Columbus, $\mathrm{OH}$ 43210, USA.

Received: 21 March 2020 Accepted: 16 July 2020

Published online: 07 September 2020

\section{References}

1. Cammack KM, Austin KJ, Lamberson WR, Conant GC, Cunningham HC. RUMINANT NUTRITION SYMPOSIUM: Tiny but mighty: the role of the rumen microbes in livestock production. J Anim Sci 2018;96(10):4481-4481.

2. Loor JJ, Elolimy AA, McCann JC. Dietary impacts on rumen microbiota in beef and dairy production. Anim Front. 2016;6(3):22-9.

3. Wolff SM, Ellison MJ, Hao Y, Cockrum RR, Austin KJ, Baraboo M, et al. Diet shifts provoke complex and variable changes in the metabolic networks of the ruminal microbiome. Microbiome. 2017;5:60.

4. Kleinschmit DH, Anderson JL, Schingoethe DJ, Kalscheur KF, Hippen AR. Ruminal and intestinal degradability of distillers grains plus solubles varies by source. J Dairy Sci. 2007;90(6):2909-18.

5. Shen JS, Chen YY, Moraes LE, Yu ZT, Zhu WY. Effects of dietary protein sources and nisin on rumen fermentation, nutrient digestion, plasma metabolites, nitrogen utilization, and growth performance in growing lambs. J Anim Sci. 2018;96(5):1929-38.

6. Held J: Feeding soy hulls and dried distillers grain with solubles to sheep. South Dakota State University, Extension Extra Archives. 2006. http:// openprairie.sdstate.edu/extension_extra/75. Accessed 1 Jan 2006.

7. Klopfenstein TJ, Erickson GE, Bremer VR. BOARD-INVITED REVIEW: Use of distillers by-products in the beef cattle feeding industry. J Anim Sci 2008; 86(5):1223-1231.

8. Schingoethe DJ, Kalscheur KF, Hippen AR, Garcia AD. Invited review: the use of distillers products in dairy cattle diets. J Dairy Sci. 2009;92(12):5802-13.

9. Callaway TR, Dowd SE, Edrington TS, Anderson RC, Krueger N, Bauer N, et al. Evaluation of bacterial diversity in the rumen and feces of cattle fed different levels of dried distillers grains plus solubles using bacterial tagencoded FLX amplicon pyrosequencing. J Anim Sci. 2010;88(12):3977-83.

10. Aldai N, Klieve AV, Dugan MER, Kramer JKG, Ouwerkerk D, Aalhus JL, et al. Evaluation of rumen fatty acid hydrogenation intermediates and differences in bacterial communities after feeding wheat- or corn-based dried distillers grains to feedlot cattle. J Anim Sci. 2012;90(8):2699-709.

11. Castillo-Lopez E, Ramirez Ramirez HA, Klopfenstein TJ, Anderson CL, Aluthge ND, Fernando SC, et al. Effect of feeding dried distillers grains with solubles on ruminal biohydrogenation, intestinal fatty acid profile, and gut microbial diversity evaluated through DNA pyro-sequencing. J Anim Sci. 2014;92(2):733-43.

12. Ramirez HAR, Nestor K, Tedeschi LO, Callaway TR, Dowd SE, Fernando SC, et al. The effect of brown midrib corn silage and dried distillers' grains with solubles on milk production, nitrogen utilization and microbial community structure in dairy cows. Can J Anim Sci. 2012;92(3):365-80.

13. Castillo-Lopez E, Jenkins CJR, Aluthge ND, Tom W, Kononoff PJ, Fernando SC. The effect of regular or reduced-fat distillers grains with solubles on rumen methanogenesis and the rumen bacterial community. J Appl Microbiol. 2017;123(6):1381-95.

14. Cheng KJ, McCowan RP, Costerton JW. Adherent epithelial bacteria in ruminants and their roles in digestive tract. Am J Clin Nutr. 1979:32(1):139-48.

15. Mulder TD, Goossens K, Peiren N, Vandaele L, Haegeman A, Tender CD, et al. Exploring the methanogen and bacterial communities of rumen environments: solid adherent, fluid and epimural. FEMS Microbiol Ecol. 2017; 93(3):fiw251. https://doi.org/10.1093/femsec/fiw251.

16. Cho SJ, Cho KM, Shin EC, Lim WJ, Hong SY, Choi BR, et al. 16S rDNA analysis of bacterial diversity in three fractions of cow rumen. J Microbiol Biotechnol. 2006;16(1):92-101.

17. Sadet $\mathrm{S}$, Martin C, Meunier B, Morgavi DP. PCR-DGGE analysis reveals a distinct diversity in the bacterial population attached to the rumen epithelium. Animal. 2007;1 (7):939-44. 
18. Scharen M, Kiri K, Riede S, Gardener M, Meyer U, Hummel J, et al. Alterations in the rumen liquid-, particle- and epithelium-associated microbiota of dairy cows during the transition from a silage- and concentrate-based ration to pasture in spring. Front Microbiol. 2017:8:744.

19. Mao SY, Zhang ML, Liu JH, Zhu WY. Characterising the bacterial microbiota across the gastrointestinal tracts of dairy cattle: membership and potential function. Sci Rep. 2015;5:16116.

20. Chen YH, Oba M, Guan LL. Variation of bacterial communities and expression of toll-like receptor genes in the rumen of steers differing in susceptibility to subacute ruminal acidosis. Vet Microbiol. 2012;159(3-4): 451-9.

21. Li MJ, Zhou M, Adamowicz E, Basarab JA, Guan LL. Characterization of bovine ruminal epithelial bacterial communities using 16S rRNA sequencing, PCRDGGE, and qRT-PCR analysis. Vet Microbiol. 2012;155(1):72-80.

22. Malmuthuge N, Li MJ, Chen YH, Fries P, Griebel PJ, Baurhoo B, et al. Distinct commensal bacteria associated with ingesta and mucosal epithelium in the gastrointestinal tracts of calves and chickens. FEMS Microbiol Ecol. 2012; 79(2):337-47.

23. Pitta DW, Pinchak WE, Dowd SE, Osterstock J, Gontcharova V, Youn E, et al, Rumen bacterial diversity dynamics associated with changing from Bermudagrass hay to grazed winter wheat diets. Microb Ecol. 2010;59(3): 511-22.

24. Liu JH, Zhang ML, Zhang RY, Zhu WY, Mao SY. Comparative studies of the composition of bacterial microbiota associated with the ruminal content, ruminal epithelium and in the faeces of lactating dairy cows. Microb Biotechnol. 2016;9(2):257-68.

25. Ren QM, Si HZ, Yan XT, Liu C, Ding LM, Long RJ, et al. Bacterial communities in the solid, liquid, dorsal, and ventral epithelium fractions of yak (Bos grunniens) rumen. Microbiologyopen. 2020;9(2):e963

26. Li ZP, Mu CL, Xu YX, Shen JS, Zhu WY. Changes in the solid-, liquid-, and epithelium-associated bacterial communities in the rumen of hu lambs in response to dietary urea supplementation. Front Microbiol. 2020;11:244.

27. Huws SA, Creevey CJ, Oyama LB, Mizrahi I, Denman SE, Popova M, et al. Addressing global ruminant agricultural challenges through understanding the rumen microbiome: past, present, and future. Front Microbiol. 2018;9:2161.

28. Dai ZL, Zhang J, Wu GY, Zhu WY. Utilization of amino acids by bacteria from the pig small intestine. Amino Acids. 2010;39(5):1201-15.

29. Caporaso JG, Kuczynski J, Stombaugh J, Bittinger K, Bushman FD, Costello EK, et al. QIIME allows analysis of high-throughput community sequencing data. Nat Methods. 2010;7(5):335-6.

30. Edgar RC, Haas BJ, Clemente JC, Quince C, Knight R. UCHIME improves sensitivity and speed of chimera detection. Bioinformatics. 2011;27(16): 2194-200.

31. Quast C, Pruesse E, Yilmaz P, Gerken J, Schweer T, Yarza P, et al. The SILVA ribosomal RNA gene database project: improved data processing and webbased tools. Nucleic Acids Res. 2013;41:D590-6.

32. Price MN, Dehal PS, Arkin AP. FastTree: computing large minimum evolution trees with profiles instead of a distance matrix. Mol Biol Evol. 2009;26(7):1641-50.

33. Asshauer KP, Wemheuer B, Daniel R, Meinicke P. Tax4Fun: predicting functional profiles from metagenomic 165 rRNA data. Bioinformatics. 2015; 31(17):2882-4

34. Denman SE, MCSweeney CS. Development of a real-time PCR assay for monitoring anaerobic fungal and cellulolytic bacterial populations within the rumen. FEMS Microbiol Ecol. 2006;58(3):572-82.

35. Sylvester JT, Karnati SKR, Yu ZT, Morrison M, Firkins JL. Development of an assay to quantify rumen ciliate protozoal biomass in cows using real-time PCR. J Nutr. 2004;134(12):3378-84.

36. Denman SE, Tomkins NW, MCSweeney CS. Quantitation and diversity analysis of ruminal methanogenic populations in response to the antimethanogenic compound bromochloromethane. FEMS Microbiol Ecol. 2007;62(3):313-22.

37. Devkota S, Wang YW, Musch MW, Leone V, Fehlner-Peach H, Nadimpalli A, et al. Dietary-fat-induced taurocholic acid promotes pathobiont expansion and colitis in II10(-/-) mice. Nature. 2012;487(7405):104-8.

38. Williams AG, Coleman GS. The rumen protozoa. In: Hobson PN, Stewart CS, editors. The rumen microbial ecosystem. NY: Springer; 1997. p. 73-139.

39. Firkins $\mathrm{J}, \mathrm{Y} u$ Z, Morrison M. Ruminal nitrogen metabolism: perspectives for integration of microbiology and nutrition for dairy. J Dairy Sci. 2007; 90(Suppl 1):E1-16.

40. Newbold CJ, de la Fuente G, Belanche A, Ramos-Morales E, McEwan NR. The role of ciliate protozoa in the rumen. Front Microbiol. 2015:6:1313.
41. McAllister TA, Bae HD, Jones GA, Cheng KJ. Microbial attachment and feed digestion in the rumen. J Anim Sci. 1994:72(11):3004-18.

42. Krause DO, Denman SE, Mackie RI, Morrison M, Rae AL, Attwood GT, et al. Opportunities to improve fiber degradation in the rumen: microbiology, ecology, and genomics. FEMS Microbiol Rev. 2003;27(5):663-93.

43. Edwards JE, Forster RJ, Callaghan TM, Dollhofer V, Dagar SS, Cheng Y, et al. PCR and omics based techniques to study the diversity, ecology and biology of anaerobic fungi: insights, challenges and opportunities. Front Microbiol. 2017;8:1657.

44. Jeyanathan J, Martin C, Morgavi DP. The use of direct-fed microbials for mitigation of ruminant methane emissions: a review. Animal. 2014;8(2):25061.

45. Choudhury PK, Salem AZM, Jena R, Kumar S, Sigh R, and Puniya AK. Rumen microbiology: an overview In: Puniya AK, Singh R, Kamra DN, editors. The Rumen microbiology: from evolution to revolution. New Delhi: Springer. 2015. p. 3-16.

46. Sadet-Bourgeteau S, Martin C, Morgavi DP. Bacterial diversity dynamics in rumen epithelium of wethers fed forage and mixed concentrate forage diets. Vet Microbiol. 2010;146(1-2):98-104.

47. Liu JH, Bian GR, Zhu WY, Mao SY. High-grain feeding causes strong shifts in ruminal epithelial bacterial community and expression of toll-like receptor genes in goats. Front Microbiol. 2015;6:167.

48. Jiao JZ, Huang JY, Zhou CS, Tan ZL. Taxonomic identification of ruminal epithelial bacterial diversity during rumen development in goats. App Environ Microb. 2015;81(10):3502-9.

49. Liu J, Bian G, Sun D, Zhu W, Mao S. Starter feeding altered ruminal epithelial bacterial communities and some key immune-related genes' expression before weaning in lambs. J Anim Sci. 2017;95(2):910-21.

50. Vos P, Garrity G, Jones D, Krieg NR, Ludwig W, Rainey FA, et al. Bergey's manual of systematic bacteriology. Vol 3, The Firmicutes New York, NY: Springer Science \& Business Media; 2009.

51. Attene-Ramos MS, Wagner ED, Plewa MJ, Gaskins HR. Evidence that hydrogen sulfide is a genotoxic agent. Mol Cancer Res. 2006:4(1):9-14

52. Yang G. Hydrogen sulfide in cell survival: a double-edged sword. Expert Rev Clin Pharmacol. 2011;4(1):33-47.

53. Stewart CS, Flint HJ, Bryant MP. The rumen bacteria. In: Hobson PN, Stewart CS, editors. The rumen microbial ecosystem. NY: Springer; 1997. p. 10-72.

54. Christl SU, Eisner HD, Dusel G, Kasper H, Scheppach W. Antagonistic effects of sulfide and butyrate on proliferation of colonic mucosa: a potential role for these agents in the pathogenesis of ulcerative colitis. Digest Dis Sci. 1996:41(12):2477-81.

55. Mann E, Wetzels SU, Wagner M, Zebeli Q, Schmitz-Esser S.

Metatranscriptome sequencing reveals insights into the gene expression and functional potential of rumen wall bacteria. Front Microbiol. 2018:9:43.

Ready to submit your research? Choose BMC and benefit from:

- fast, convenient online submission

- thorough peer review by experienced researchers in your field

- rapid publication on acceptance

- support for research data, including large and complex data types

- gold Open Access which fosters wider collaboration and increased citations

- maximum visibility for your research: over $100 \mathrm{M}$ website views per year

At $\mathrm{BMC}$, research is always in progress.

Learn more biomedcentral.com/submissions 